\title{
Fuzzy Logic Based Safety Measures for Vehicles on Road
}

\author{
Rezuana Bai $\mathbf{J}^{1}$, Premson $\mathbf{Y}^{2}$ \\ Assistant Professor, Dept. of Electronics and Communication Engineering, Govt.RIT, Kottayam ${ }^{1,2}$
}

\begin{abstract}
Safety of vehicles moving at high speeds on the road is a matter of big concern. Fuzzy logic based systems could be incorporated in vehicles for this purpose. A fuzzy logic based model is developed for good vehicle control. Image or echo based techniques can be used to get the required inputs for the model. The system is developed such that, when the vehicle moves close to the left or right line on the road, the steering is turned to bring the vehicle to the middle of the road. The break force is decided based on the speed of the vehicle and its distance from the front vehicle. These requirements are best modelled by the Fuzzy logic method. When the vehicle goes out of its intended path or when it is excessively closer to any vehicle or other objects, this system can act with the artificial intelligence capabilities to avoid possible accidents.
\end{abstract}

Keywords: Fuzzy logic, Defuzzification, Membership Functions, Rule-base, Degree of Membership.

\section{INTRODUCTION}

More than one million people die every year in road values required on the process based on the 'rule base' and accidents. Most of these accidents are attributed to careless the 'Dufuzzification' is the process of obtaining crisp driving. Artificial intelligence can play a crucial role in values for the output variables from the fuzzy or vague reducing the number of accidents this paper investigates input values. The calculated outputs are the force required the methods that can be incorporated in vehicles in an on the break and turn needed for the steering to control the emergency situation. When the vehicle moves very close vehicle [1].

to another vehicle or object at certain speeds, a fuzzy logic based system can take control of the vehicle. Also when the vehicle goes out of the specified track unintentionally the system can bring it to the middle of the track.

Fuzzy logic models the human thinking process. Correct decisions are taken from vague or unclear inputs. Fig. 1 shows the general Fuzzy logic system block diagram. The 'process' represents the vehicles movements. $\mathrm{r}(\mathrm{t})$ represents the parameters for the vehicles movement like acceleration and steering turn. The 'measurement' includes measuring of the distance of the vehicle from the left and right lines of the track, the speed and the distance from the front line. The 'decision making logic' calculates the correction

\section{THE FUZZY SYSTEM DEVELOPMENT}

A. The Input and outputs

There are four inputs for the proposed model. Two distances from the side lines is assumed and named as 'leftdistance' and 'rightdistance'. This distance can be calculated using image processing techniques. Images can be obtained from two cameras connected at the left and right of the vehicle. Even one image using a front camera can also be used for getting all the measurements for this work. In this paper these distances are assumed as the distances measured from the images. So the number of pixels is used in the input range display. A range of 0 to 250 is assigned for this variable.

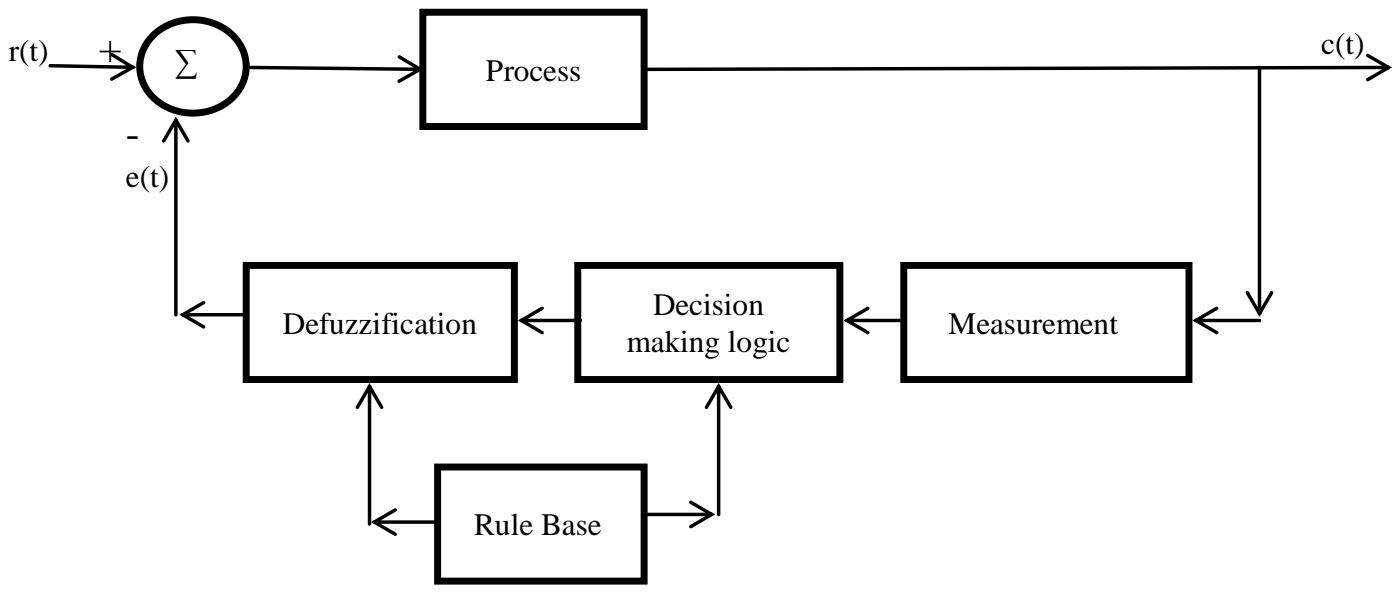

Fig.1. Fuzzy Logic Based System 

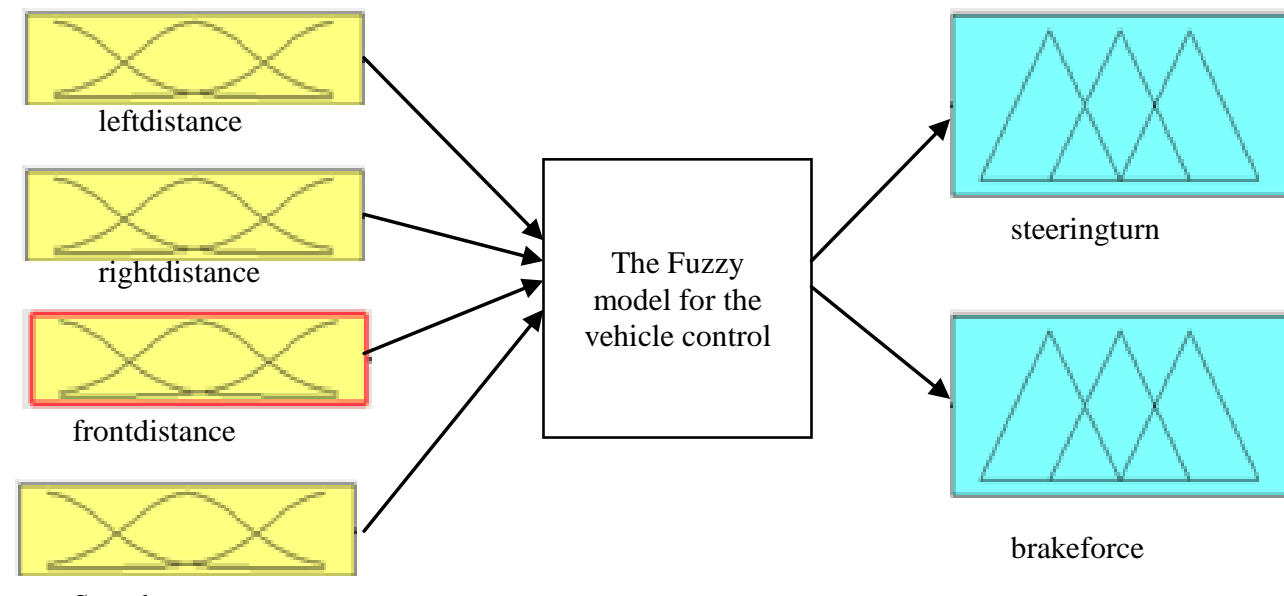

Speed

brakeforce

Fig.2. Fuzzy model with inputs(left side) and outputs(right side)

Another distance measured from the front vehicle or any obstacle is assumed as the third input, 'frontdistance'. In this paper this distance is taken as the number of pixels from an image. So a range of 0 to 300 (pixels) is assumed. The fourth input is the 'speed' and a range of $0-150 \mathrm{~km} / \mathrm{s}$ is assumed in the design. Fig. 2 shows the inputs and outputs for this model.

The outputs for this design are the 'steeringturn' and the 'brakeforce'. The first represents the angle to be turned by the steering when the vehicle starts moving towards any side. Immediately the system should identify that and be able to bring under control. The second represents the force to be applied in the brake system depending upon various situations like, distance from the front vehicle being very close, or the speed being very high etc. All these are determined by defining the rule base in the fuzzy logic design $[2,3,5]$.

\section{B. The Membership Functions}

The Membership functions used for the 'leftdistance' are 'Iclose', 'Imedium' and 'Ifar'. Fig. 3 shows these membership functions. These assume that the car is close to, at right distance and away from the left line, respectively. Similar membership variables are assumed for the 'rightdistance' and 'frontdistance'. The membership variables assumed for 'speed' are 'slow', 'medium' and 'fast'.

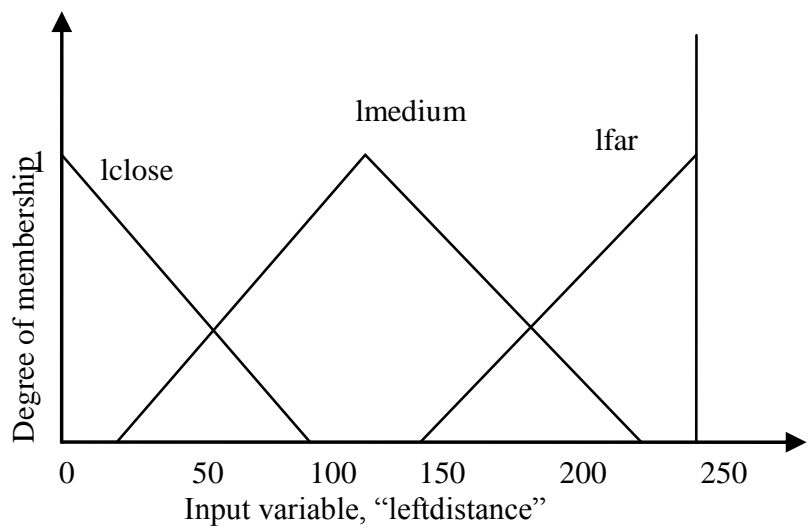

Fig3. Membership Functions for Input Variable 'leftdistance'
The membership functions for the output variable 'steeringturn' are 'turnleft', 'gostraight' and 'turnright'(Fig.4). Membership functions for 'brakeforce' are 'lowbrake', mediumbrake' and 'hardbrake' (Fig. 5).

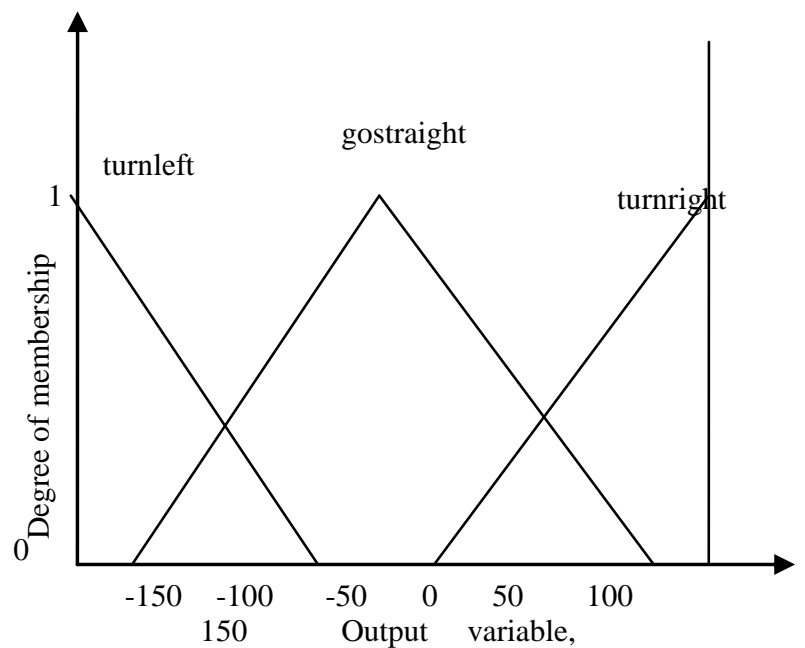

Fig.4. Membership Functions for Output Variable 'steeringturn'

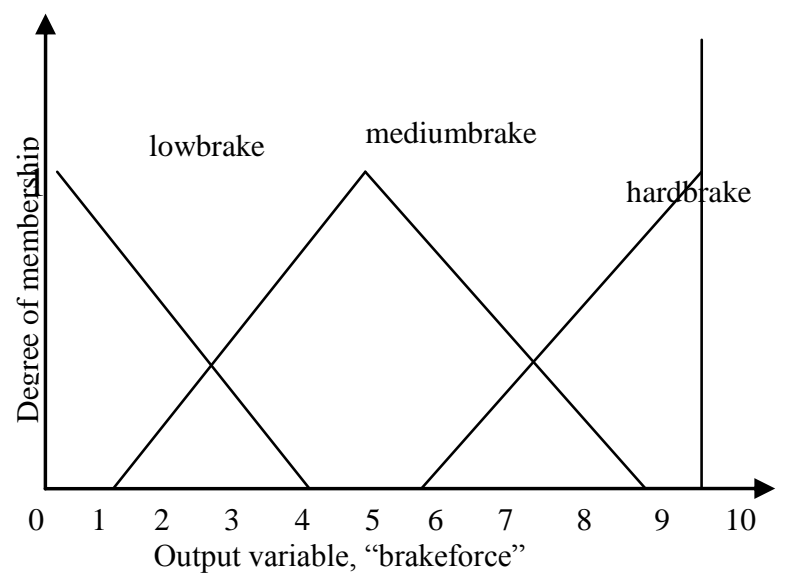

Fig.5. Membership Functions for Output Variable 'brakeforce'

C. The rule base

The rule base in fuzzy is defined as a set of if-then statements. Table 1 shows the rule base used in this work. 


\section{TABLE I RULE BASE FOR THE FUZZY MODEL}
1. If (leftdistance is lclose) then (steering turn is turnright)
2. If (leftdistance is medium) then (steering turn is gostraight)
3. If (leftdistance is lfar) then (steering turn is turnleft)
4. If (frontdistance is fclose) and (speed is slow) then (breakforce is mediumbreak)
5. If (frontdistance is fmedium) and (speed is slow) then (breakforce is lowbreak)
6. If (frontdistance is ffar) and (speed is slow) then (breakforce is lowbreak)
7. If (frontdistance is fclose) and (speed is medium) then (breakforce is hardbreak)
8. If (frontdistance is fmedium) and (speed is medium) then (breakforce is mediumbreak)
9. If (frontdistance is ffar) and (speed is medium) then (breakforce is lowbreak)
10. If (frontdistance is fclose) and (speed is fast) then (breakforce is hardbreak)
11. If (frontdistance is fmedium) and (speed is fast) then (breakforce is mediumbreak)
12. If (frontdistance is ffar) and (speed is fast) then (breakforce is lowbreak)
13. If (rightdistance is rclose) then (steeringturn is turnleft)
14. If (rightdistance is rmedium) then (steeringturn is gostraight)
15. If (rightdistance is rfar) then (steeringturn is turnright)

\section{III.RESULTS AND DISCUSSIONS}

The running of the fuzzy model is shown as the screenshot, in Fig. 6. 'readfis' and 'evalfis' functions in MATLAB are used for this purpose [6]. The readfis reads the fuzzy model, 'car' and stores it to a variable called 'fis'. This variable is used in the 'evalfis' function along with inputs for finding output of the system. The inputs given in the first case are [0 250300 40]. These are the 'leftdistance', 'rightdistance', 'frontdistance' and the 'speed'.

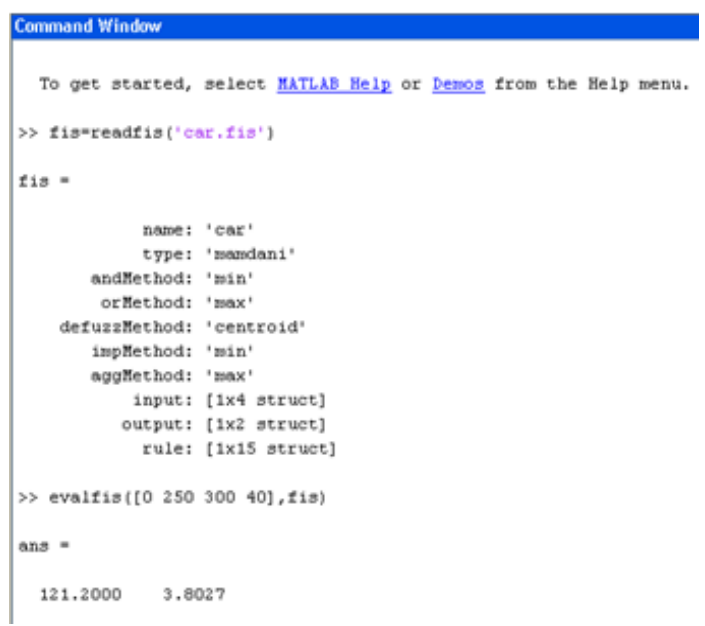

Fig. 6 Screen Shot of Fuzzy Model Evaluation

TABLE II-A INPUTS AND OUTPUTS FOR THE FUZZY MODEL (CASE-1)

\begin{tabular}{|c|c|c|c|}
\hline \multirow{3}{*}{ inputs } & \multicolumn{3}{|c|}{ Case(1) } \\
\hline & \multirow[b]{2}{*}{$\begin{array}{l}\text { Input } \\
\text { values }\end{array}$} & \multicolumn{2}{|c|}{ outputs } \\
\hline & & $\begin{array}{l}\text { steering } \\
\text { turn }\end{array}$ & $\begin{array}{l}\text { brake } \\
\text { force }\end{array}$ \\
\hline leftdistance & 0 & \multirow{4}{*}{121.2} & \multirow{4}{*}{3.8027} \\
\hline rightdistance & 250 & & \\
\hline frontdistance & 300 & & \\
\hline speed & 40 & & \\
\hline
\end{tabular}

Tables 2-A\&B shows the inputs and outputs for three cases. The distances are in terms of the number of pixels as measured form road image. The speed is in $\mathrm{km} / \mathrm{hr}$. The 'steeringturn' is in degrees. 0 indicating no turn, -180 indicating maximum left turn +180 , maximum right turn.
The 'brakeforce' is assumed an arbitrary scale of 0 to 10.0 for no braking and 10 for the maximum braking.

TABLE II-B INPUTS AND OUTPUTS FOR THE FUZZY MODEL (CASE-2 \&3)

\begin{tabular}{|c|c|c|c|c|c|}
\hline \multicolumn{3}{|c|}{ Case(2) } & \multicolumn{3}{|c|}{ Case(3) } \\
\hline \multirow{2}{*}{$\begin{array}{l}\text { Input } \\
\text { value } \\
\text { s }\end{array}$} & \multicolumn{2}{|c|}{ output } & \multirow{2}{*}{$\begin{array}{l}\text { Input } \\
\text { value } \\
\text { s }\end{array}$} & \multicolumn{2}{|c|}{ output } \\
\hline & $\begin{array}{l}\text { steering } \\
\text { turn }\end{array}$ & $\begin{array}{l}\text { brakef } \\
\text { orce }\end{array}$ & & $\begin{array}{l}\text { steerin } \\
\text { gturn }\end{array}$ & $\begin{array}{l}\text { break } \\
\text { force }\end{array}$ \\
\hline 125 & \multirow{4}{*}{0.0} & \multirow{4}{*}{8.6783} & 125 & \multirow{4}{*}{0.0} & \multirow{4}{*}{6.4568} \\
\hline 125 & & & 125 & & \\
\hline 25 & & & 100 & & \\
\hline 140 & & & 140 & & \\
\hline
\end{tabular}

Case(1) gives outputs of, 'steeringturn' $=121.2^{0}$ turn and 'brakeforce' $=3.8027$. This indicates as the car is close to the left line it should turn right. Since the speed is low it needs only a low braking.

In Case(2) of execution it can be seen that the force on brake is 8.6783 and turn of steering as $0^{0}$. This is because the speed became 140 . So it should slow down with a hard braking. Also in this case the vehicle is close to the front vehicle or object. And since the distances from the sidelines are equal, there is no need to turn the steering. In Case(3), the force is coming as 6.4568. In this case the front distance has increased to 100 instead of 25 in the previous case. So a hard braking is not needed.

Since the images are taken when the vehicle is moving, motion filters available in image processing techniques may be used for processing the images. The basic idea in these sorts of filters is to subtract successive images so that the suddenly changing background can be removed from the resulting image retaining the wanted information (the lines in this case).

The fuzzy logic system is inherently adaptive in nature. Instead of using predefined fixed speeds or steering turns, these values are decided from the measurements. So the environment determines the actual speed and turn of the vehicle.

Their needs some discussions on the timing issues of the system. The time needed to control before a collision depends upon the sensing devices, the processing system and the actuation mechanisms. There are fuzzy controllers 
and associated softwares which support a high speed processing [1]. The sensing system can have a redundant arrangement of IR LED photodiode combination or sonar based systems along with the imaging devices. The actuation mechanism can be motor controlled. A proportionate voltage generated by the system can be used to control the motor drive. Since the system is adaptive at high speeds the break system is actuated for a long distance from the front vehicle. This reduces the needs of very high timing constraints. But it is crucial when suddenly some other vehicle comes in front of our vehicle.

\section{IV.CONCLUSION}

This paper is an attempt to improve the safety measures in vehicles on road. There are lots of issues related with the ideas presented in this paper. One of them is, when the fuzzy system should take control of the vehicle. When the driver wanted to intentionally change the track, there need the mechanism to deactivate the fuzzy system. The fuzzy system presented here is useful in the event of sleepy driving and mainly to avoid collisions with other vehicles. Image processing based detection is assumed in the development of this model. Other methods like sonar echo methods or light reflection methods also used. Redundant methods can improve the robustness of the system.

\section{REFERENCES}

[1] J. E. Naranjo and C. Gonzalez, "Adaptive Fuzzy Control for InterVehicle Gap Keeping”, IEEE Trans. Intell. Transp. Syst. Vol.4, No.3,pp 132-142, Sept. 2003

[2] M.Cao and E. Hall, "Fuzzy Logic Control for an Automated Guided Vehicle", Research paper, Centre for Robotic Reasearch, University of Cincinnati, 2003

[3] Anthony B. Wil,1 "Intelligent Vehicle Braking and Steering Control Systems". Master's Thesis, Purdue University, 1997

[4] Timothy J. Ross, "Fuzzy logic with Engineering applications", McGraw-Hill, 1997

[5] Behavior-Decision Fuzzy Algorithm. Proc. IEEE International Conference on Robotics and Automation, pp. 117-122.

[6] Mathworks, Web Pages

\section{BIOGRAPHIES}

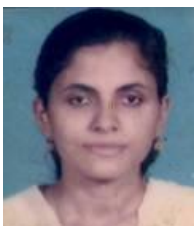

Mrs. Rezuana Bai $\mathbf{J}$ is a holder of Masters Degree in Control and Instrumentation from IIT Madras, India. She has 16 years of teaching experience in India and Oman. She is the winner of the best hardware project award from the Electrical department of IIT Madras (2005). Her areas of research include control engineering, Fuzzy Logic, instrumentation etc. Currently she is working as Assistant Professor, Dept. of Electronics and Communication Engineering, Govt.RIT, Kottayam

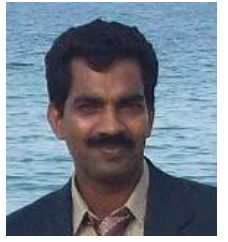

Mr. Premson $\mathbf{Y}$ is having M.Tech in Microelectronics and VLSI design from the Electrical Engineering dept. of IIT Madras, India. $\mathrm{He}$ has 20 years of teaching experience in India and Oman. His areas of research include Digital Signal Processing, Image Processing, Fuzzy Logic etc. Currently he is working as Assistant Professor, Dept. of Electronics and Communication Engineering, Govt.RIT, Kottayam. 